\title{
The Finnish database on drug utilisation
}

\author{
Timo Klaukka \\ The Social Insurance Institution, PB 450, FIN-00101 Helsinki, Finland \\ timo.klaukka@kela.memonet.fin
}

\begin{abstract}
This article describes main sources of data used to study drug utilisation in Finland. National health interview studies have been used for this since 1964. Sales statistics are based on information from wholesalers, and the Nordic ATC/DDD methodology is availed in order to make the numbers comparable with the other countries. The most important database is a national prescription register, which exists since 1994 and includes all reimbursed medication purchases. The data were originally collected for administrative purposes, but they provide useful material also for pharmaco-epidemiological and -economic research.
\end{abstract}

Key words: Drug utilisation, prescription register, health interview surveys, sales statistics

Medicines are one of the most frequently used treatments of a majority of diseases and complaints. Insulin and antibiotics, for instance, can be life-saving, and modern surgery techniques, organ transplantations and other similar treatments were not applicable without anaesthetics, analgesics or immunosuppressants. Medicines can, however, cause also adverse effects, and they always create costs. If medicines are used inappropriately, all the negative aspects are unnecessary and avoidable.

Costs of medicines are increasing by 7-10\% annually in most of the European countries. During a decade, the share of medicines of the total health care expenditure in Finland has risen from 9\% to the present $15 \%$. This is not due to an increasing number of users: in two consecutive health interview surveys in 1987 and 1995/96 the proportion of medicine users of the total population was the same, around $40 \%$, at the time of the interview (1). The main reason for the continuous growth of the costs is the replacement of older medicines by newer and more expensive ones (2).

Forty percent of the population using prescription medicines is as such worrying. Medicines have become one of most frequently used products in the Western societies. Their effects are only rarely curative; much more of the medications aim at relieving symptoms rather than preventing from diseases. A substantial part is used for the treatment or prophylaxis of life-style related diseases. These include hypertension, type II diabetes, cardiac insufficiency, coronary heart disease, insomnia and chronic obstructive pulmonary disease, for which there were also nonpharmacological therapies available.

For these reasons, information on frequency and distribution about use of medicines in the population is important. We need to know, for instance, the proportion of the population exposed to medicines in general and to various groups of them in particular. Multiusers of medicines should be identified in order to improve drug safety, and population groups with larger than average medicine costs should be found to strengthen reimbursement systems. Information must be gathered also about the quality of care of major diseases.

\section{THREE MAIN SOURCES OF DATA}

Central elements of the Finnish database on drug utilisation derive from three major sources:

1. Population-based national health interview surveys

2. Sales statistics

3. A national prescription register

Additional data collecting methods are, for instance, regional or local studies on defined patient or other population groups, statistics on trade of pharmaceuticals, and the number of prescriptions purchased in pharmacies.

\section{HEALTH INTERVIEW SURVEYS}

Finland has conducted national health interview surveys since 1964 (1). The first study was done just before the general sickness insurance act came into force, and a follow-up study four years later evaluated the effects of the innovation on health services use, health status and the costs of the use to the families (3). Later studies have been conducted in 1976, 1987 and 1995/96. Main indicators and questions have been the same in most or all of the studies in order to make the comparison of the results possible.

Use of medicines has been studied in all these surveys. The information from the two first studies was on a rather general level, but in 1976 the brand names of prescription medicines used by the interviewee were recorded for the first time (4), and in the $1995 / 96$ study this method was applied also for OTC and herbal medicines. 
A major advantage of studying use of medicines in health interview surveys is that the users can be categorised by various background factors, such as age, gender, place of residence, income, education and chronic morbidity. Sample sizes, however, are for practical reasons mostly relatively small, and thus information about rarely used medicines remains scarce. An additional problem is that the reference period of the use cannot be long, because taking a tablet or a pill is not so extraordinary that everybody could remember it very much later. This diminishes further the number of 'cases' found in an interview study.

At the moment, WHO-EURO is constructing a standardised set of questions about use of medicines (and several other indicators as well) to be included in national health interview surveys. This will provide countries with an opportunity to get a commonly accepted and tested series of questions, and the results will be comparable between all the countries which are applying the same methodology.

\section{SALES STATISTICS ON MEDICINES}

The Nordic countries are in a unique position concerning statistics on the consumption of medicines. Since the late 1970 s, a common classification of medicines (ATC) as well as a standardised unit of measurement (defined daily dose, DDD) has been availed in all these countries. Relatively long time trends in the consumption can thus be investigated, and use of various groups of medicines can be compared within the Nordic area.

Nordic Statistics on Medicines was published for the first time in 1979 concerning the years 1975-1977 (5). Not all medicine groups were included, but the coverage went wider in the later books, which were published on regular intervals until 1996 (6). Since then no common publication has appeared, but the individual countries have continued to publish their national statistics using the traditional methodology. Thus the comparison is still possible, although one has to search for the figures from separate books, which are not structured in an identical way.

The Finnish Statistics on Medicines was published for the first time in 1988, and the figures presented concerned the year 1987 (7). Before that, figures were calculated manually and published solely in the Nordic books. Since 1988, the two-language (Finnish and English) book has been produced every year. In addition to ATC/DDD based sales statistics, the report contains administrative information about pharmaceutical care, as well as figures on drug expenditures derived from the national reimbursement system.

Sales statistics are a convenient source to study time trends and regional variations in drug consumption. One of the major problems with the data is that they do reveal facts neither about the users, nor about the prescribers.

\section{REGISTERS}

The Finnish patients get ordinarily a fifty percent compensation of their medication costs (of the sum above the fixed deductible FIM50). There are also two special categories, the lower (75\%) and the higher $(100 \%)$. Around 40 diseases (diabetes, epilepsy, psychoses, cancer, glaucoma, etc.) belong to the higher, and more than 10 diseases (hypertension, asthma, cardiac insufficiency, rheumatoid arthritis, etc.) to the lower of the special categories. These diseases are defined by the Cabinet. The Ministry of Social Affairs and Health decides which substances can be included in the special reimbursement categories in the treatment of the diseases concerned.

From a pharmaco-epidemiological point of view it is most important, that in order to get an entitlement to special reimbursement, the patient must apply for it from the Social Insurance Institution (SII). For this she/he must attach a certificate from a doctor (mostly a specialist) verifying the diagnosis and severity of the disease. If criteria set by the SII are met, the application will be granted, and the identification code of the patient is registered centrally by the SII, and updated regularly. This means that we have individual-based patient registers of nearly all major diseases, starting from the late 1960s.

These registers can be used for research purposes, for instance for drawing samples for postal questionnaire studies, and for studying the incidence and prevalence as well as trends of the diseases, and their medication costs.

Some examples: at the end of 1999, hypertension was by far the largest disease in the register: around 455,000 patients were included (the total population size is 5,1 million). Asthma is now the second in order with 185,000 registered patients, and coronary heart disease the third $(172,000)$. Figures on all these and other diseases are regularly published in the Finnish Statistics on Medicines. The book contains also information about average medication costs per patient during the statistical year. For instance, in 1999 the costs of medicines for hypertension reimbursed in the special category amounted to FIM 1,652, and they were FIM 2,513 for asthma, FIM 4,936 for Parkinson's disease, but only FIM 137 for hypothyreosis (2).

Regionally the medication costs of various diseases may vary substantially. The highest cost per patient are above the lowest one by FIM 205 for hypertension, FIM 605 for asthma, and as much as FIM 1,149 for rheumatoid arthritis. Åland islands have become well known for their relatively low cost of medication (8).

\section{PRESCRIPTION REGISTER}

The history of the Finnish prescription register originates from an administrative need for information about the patients' medication. The Finnish drug reim- 
bursement system includes an annual maximum which the patient must pay him/herself for reimbursable medicines. In 2000, this sum was FIM 3,340. When the patient's out-of-pocket costs reach this level, he/she can get free medicines (reimbursable and necessary) for the remaining part of the calendar year. But not automatically: the local bureau of the SII must first investigate that all the medications purchased by the patient seem to have been necessary. To make such an investigation possible, a computerized central register on all reimbursed medications was established in 1994. The experts in the bureaus can get all reimbursed purchases of an individual on the screen. If, for instance, large amounts of psychotropic medicines clearly dominate the view, the entitlement to free medicines cannot be granted without further inquiries about the real need for such a medication.

Thus the national prescription register originated for commonly acceptable administrative reasons, not primarily for research purposes. The register would perhaps not exist if the latter had been the main aim. But because information about the prescribing of medicines is very important for several reasons, also researchers have become regular users of this databank.

The information of the register is collected monthly from all pharmacies. The register includes around 25 million prescriptions annually, and the data concern all what is on the prescription form, i.e. the patient, the medicine, the doctor, and costs and reimbursements. Also dates of the prescribing and purchasing are included. By linking the identification code of the patient to other registers, the place of residence of the patient as well as the speciality of the prescribing doctor can be added to the information.

Information about the purpose of the medication is scarce. Only about half of all prescriptions include any information about this, and the expressions used by the doctors vary a lot, which makes it difficult to code them in a standardised way. Here comes the special reimbursement system to assist: if the patient is entitled for special reimbursement of the medicines which he/she is purchasing, the pharmacy records the code number to the databank. Thus it is possible to have a closer look at the medical treatment of these diseases. For other complaints, this can be done only if the group of medicines concerned are solely or mostly used for a specific disease.

Some purchases of medicines remain outside of the register. This concerns nearly all OTC-medications, and naturally also medicines, which are used only in hospitals. There are also groups of prescription medicines which do not belong to the list of reimbursable drugs. For instance, most of the small packages of hypnotics have been withdrawn from the list on the manufacturers' initiative. The reason for this is that the price can be set freely, if the preparation is outside of the system.
If a package of a medicine is relatively inexpensive (below FIM50 in the basic category), the patient does pay it totally him/herself without getting the reimbursement, and thus that sort of purchase will not be included in the register.

In principle, all researchers are welcomed to use the data of the prescription register. For research officers of the Social Insurance Institution (like myself), this goes without any special application. The confidential nature of the data and in part also the lack of resources to produce material from the register makes it necessary to restrict somewhat the access to it. Thus the researchers outside of SII must apply for the data by sending their research plan to the SII, which studies their relevance and estimates the costs which will incur when the data are processed. Data cannot be provided, if they would be used directly for commercial purposes.

The database has been a fruitful source of data for several studies. We have investigated, for instance, use of medicines among diabetic individuals - not only antidiabetics but also all other medicines which are used by this patient group (9). In comparison to demographically matched non-diabetic controls, the patients use nearly all prescription medicines more, and their total medication costs are 3.5 times as high as those of the control group.

Technical problems with the register are its size and clumsiness - the data must be purified and restructured in several ways before drivings can be started, and small computers are unable to process the data.

\section{FEEDBACK TO DOCTORS}

All Finnish doctors who have written at least 200 reimbursed prescriptions during a calendar year receive a summary of their prescriptions and their costs from the SII. This practice has been used since 1997. Each personal report is produced and mailed automatically, so that nobody else can study it without the doctor's permission. The data provided include the number of prescriptions and their distribution by the patients' age and gender, total costs of the medications, average cost of a prescription, and groups of medicines which have been most commonly prescribed by the doctor, and major groups which have caused the greatest costs. All relevant figures are compared with the average of the speciality of the doctor concerned.

Finnish doctors have taken the information with interest, and almost no negative attitudes have occurred. A problematic question is whether these summaries have somehow influenced prescription patterns. This is difficult to evaluate, because all doctors get in principle the same information, and therefore no control group exists. On the basis of the register it is also difficult to evaluate the quality of the prescribing, because there is no information available about, for instance, the severity of the disease treated. 


\section{REFERENCES}

1. Arinen S, Häkkinen U, Klaukka T, Klavus J, Lehtonen R, Aro S. Health and use of health services in Finland. Main findings of the Finnish Health Care Survey 1995/96 and changes from 1987. SVT Health Care 1998:5, Kela and Stakes, Helsinki 1998.

2. The Finnish Statistics on Medicines 1999. National Agency for Medicines and the Social Insurance Institution, Helsinki 2000.

3. Purola T, Kalimo E, Nyman K. Health services use and health status under national sickness insurance. An evaluative resurvey of Finland. Publications of the Social Insurance Institution A: 11/1974, Helsinki 1974.

4. Klaukka T, Martikainen J, Kalimo E. Drug utilization in Finland 1964-1987. Publications of the Social Insurance Institution M: 71, Helsinki 1990.

5. Nordisk läkemedelsstatistik 1975-1977. Nordiska läkemedelsnämnden, Helsinki 1979.

6. Nordic Statistics on Medicines 1993-1995. NLN publication No 43. The Nordic Council on Medicines, Uppsala 1996.

7. Suomen Lääketilasto - Finnish Statistics on Medicines 1987. National Board of Health and the Social Insurance Institution, Helsinki 1988.

8. Lahnajärvi L, Klaukka T, Enlund H. Åland - självstyrd läkemedelskonsumtion. Folkpensionsanstalten. Social trygghet och hälsa: Undersökningar 23. Helsingfors 1997.

9. Reunanen A, Martikainen J, Kangas T, Klaukka T. Nationwide survey of comorbidity, use, and costs of all medications in Finnish diabetic individuals. Diabetes Care 2000; 23: 1265-1271. 